\title{
Preliminary Exploration of Adaptive State Predictor Based Human Operator Modeling
}

\author{
Anna C. Trujillo ${ }^{1}$ and Irene M. Gregory ${ }^{2}$ \\ NASA Langley Research Center, Hampton, VA, 23681
}

\begin{abstract}
Control-theoretic modeling of the human operator dynamic behavior in manual control tasks has a long and rich history. In the last two decades, there has been a renewed interest in modeling the human operator. There has also been significant work on techniques used to identify the pilot model of a given structure. The purpose of this research is to attempt to go beyond pilot identification based on collected experimental data and to develop a predictor of pilot behavior. An experiment was conducted to quantify the effects of changing aircraft dynamics on an operator's ability to track a signal in order to eventually model a pilot adapting to changing aircraft dynamics. A gradient descent estimator and a least squares estimator with exponential forgetting used these data to predict pilot stick input. The results indicate that individual pilot characteristics and vehicle dynamics did not affect the accuracy of either estimator method to estimate pilot stick input. These methods also were able to predict pilot stick input during changing aircraft dynamics and they may have the capability to detect a change in a subject due to workload, engagement, etc., or the effects of changes in vehicle dynamics on the pilot.
\end{abstract}

\section{Nomenclature}

$\begin{array}{ll}A_{k} & =\text { amplitude } \\ \mu_{|\delta-\hat{\delta}|} & =\text { mean of the absolute difference between Stick and Stick_hat } \\ \varepsilon(t) \in R^{m} & =\text { non-parametric uncertainty in the system } \\ \phi_{k} & =\text { phase angle (rad) } \\ e\left(\theta-\theta_{c m d}\right) & =\text { Pitch }- \text { Pitch_cmd } \\ \Phi(x(t)) \in R^{N} & =\text { regressor vector of Radial Basis Functions } \\ \Theta \in R^{N x m} & =\text { unknown parameter vector in model estimation } \\ \zeta & =\text { damping ratio } \\ \tau & =\text { time delay (ms) } \\ K & =\text { gain } \\ \text { La } & =\text { zero location } \\ \text { Pitch } & =\text { vehicle pitch (deg) } \\ \text { Pitch_cmd } & =\text { commanded vehicle pitch (deg) } \\ \text { Stick_hat } & =\text { estimated stick position ([-1:1]) } \\ u(t) & =\text { pitch attitude tracking command (deg) } \\ \delta & =\text { pilot stick } \\ \theta & =\text { phitch attitude } \\ \omega & \end{array}$

\footnotetext{
${ }^{1}$ Senior Research Engineer, Crew Systems and Aviation Operations Branch, MS 152, Anna.C.Trujillo@nasa.gov, AIAA Assoc. Fellow.

${ }^{2}$ Senior Research Engineer, Dynamic Systems and Control Branch, MS 308, Irene.M.Gregory@nasa.gov, AIAA Assoc. Fellow.
} 


\section{Introduction}

$\mathrm{C}$ ontrol-theoretic modeling of the human operator dynamic behavior in manual control tasks has a long and rich history $^{1,2}$. A great deal of foundational work in understanding the human as a controller was done in the 1960's and 1970's with the development of frequency-domain based models for single-input/single-output systems by McRuer $^{3,4}$. The frequency-domain models included isomorphic structural models and behavioral based models. The classical McRuer crossover model of compensatory manual control is still used as a standard for describing the operator-vehicle behavior at the crossover frequency ${ }^{4}$. In essence, the model states that the human operator will adapt to vehicle dynamics by providing lead equalization, with an associated computational penalty, such that the combined operator-vehicle transfer function is proportional to an integrator at the crossover frequency. Another approach to understanding the human-as-the-controller behavior was the development of the optimal control model by Kleinman, Baron, and Levinson based on the assumption that a well-trained and motivated human controller behaves optimally in some sense, adjusting the pilot's compensation for a given vehicle and task, subject to human limitations ${ }^{5}$.

In the last two decades, there has been a renewed interest in modeling the human operator. Further model development by Hess in the frequency domain, a structural model ${ }^{6}$, and Schmidt in the time domain, a modified optimal control pilot model ${ }^{7,8}$, attempted to take into consideration appropriate relevant feedback that influenced a pilot's behavior such as proprioceptive cues, and visual and vestibular feedback. While these models proved useful, their application to realistic simulation tasks involved a great deal of complexity. In an attempt to simplify the model while still capturing the essential pilot behavioral characteristics, Hess proposed a simplified model that has been recently applied to systems with time-varying dynamics ${ }^{9,10}$.

In addition, to several relevant model structures to describe the pilot as a controller behavior, there has also been significant work on techniques used to identify the pilot model of a given structure ${ }^{11-17}$. These included various applications of parameter identification ranging from wavelets-based approach to frequency-based system identification techniques.

The purpose of this research is to attempt to go beyond pilot identification based on collected experimental data and to develop a predictor of short-term pilot control behavior. An experiment was conducted to quantify the effects of changing aircraft dynamics on an operator's ability to track a signal in order to eventually model a pilot adapting to changing aircraft dynamics.

\section{Procedure}

The experiment on changing aircraft dynamics is briefly summarized below. The proposed approach to pilot behavior estimator is described and two different estimation algorithms that have been applied to the problem are presented.

\section{A. Pitch Dynamics}

This research used data from an experiment that looked at short period dynamics. See Ref. 18 for a detailed description. In that experiment, longitudinal dynamics were controlled while lateral/directional and thrust values were held constant at a heading of $40 \mathrm{deg}$ and a speed of $300 \mathrm{kts}$. The altitude at the beginning of each data run was $10,000 \mathrm{ft}$. The aircraft short-period pitch attitude transfer function was:

$$
\frac{\theta}{\delta}(s)=\frac{K\left(s+L_{\alpha}\right)}{s\left[(s / \omega)^{2}+2 \varsigma(s / \omega)+1\right]} e^{-\tau s}
$$

where $\theta$ is pitch attitude and $\delta$ is pilot stick. The aircraft's shortperiod pitch attitude parameters and their associated values are listed in Table 1.

\section{Tracking Signal}

The maneuver was designed as a $30 \mathrm{sec}$ longitudinal tracking task. During the $30 \mathrm{sec}$, subjects tracked the longitudinal signal as closely as possible using a standard electronic attitude indicator. The tracking command input contained a range of frequencies and amplitudes to excite an appropriate dynamic range.

Table 1. Aircraft short-period pitch attitude parameter values.

\begin{tabular}{|l|l|}
\hline \multicolumn{1}{|c|}{ Parameter } & \multicolumn{1}{c|}{ Values } \\
\hline Gain $(K)$ & $1,2,3$ \\
\hline Zero Location $(L \alpha)$ & $0.5,1,1.5$ \\
\hline Short Period Frequency $(\omega)$ & $0.5,1,1.5 \mathrm{~Hz}$ \\
\hline Damping Ratio $(\zeta)$ & $0.4,0.7,1$ \\
\hline Time Delay $(\tau)$ & $0,75,150 \mathrm{~ms}$ \\
\hline
\end{tabular}

The longitudinal tracking task was designed to contain a variety of discrete frequencies in the frequency range associated with human pilot inputs, namely 0.1 to $10 \mathrm{rad} / \mathrm{sec}$. The input design method is described in detail by 
Klein and Morelli ${ }^{19,}{ }^{20}$. The result was the equivalent of a frequency sweep input, but used many sinusoidal components with discrete frequencies applied simultaneously, instead of a single sinusoid with frequency increasing monotonically in time.

Figure 1 shows an example tracking task. The sum-of-sines task was generated as:

$$
u(t)=\sum_{k \in\{1,2, \ldots, M\}} A_{k} \sin \left(\frac{2 \pi k t}{T}+\phi_{k}\right)
$$

where $u(t)$ is the pitch attitude tracking command (deg), the discrete frequencies $2 \pi k / T$ were chosen to span the frequency range of 0.1 to $10 \mathrm{rad} / \mathrm{sec}$, the phase angles $\left(\phi_{k}\right)$ were random, and the amplitudes $\left(A_{k}\right)$ were chosen to customize the power spectrum of the multi-sine input. In this application, the phase angles were chosen at random to emulate a random sinusoidal input. The frequency indices $(k)$ were chosen at irregular intervals, which also helped to emulate a random sinusoidal input.

\section{Subjects}

Four pilots participated as subjects. The average age was 50 (standard deviation of 3.6) years old. The average years flying was $20.5 \pm 12$ and the average number of flight hours was $3312 \pm 4483$ hrs. Subject 4 had the least number of flight hours $(<600 \mathrm{hrs})$ and years flying $(\approx 6)$ whereas the next closest subject had about 1000 flight hours and approximately 15 years of flying experience. Subject 8 had the most flight hours $(>10,000 \mathrm{hrs})$ and the most years flying ( $>30$ years) while the next nearest subject had just under 2000 flight hours.

\section{B. Pilot Behavior Representation}

The only assumption made on the structure of model of pilot control action is that the model is in the linear parameterization form:

$$
y(t)=\Theta^{T} \Phi(x(t))+\varepsilon(t)
$$

where $y(t) \in R^{m}, x(t) \in R^{n}$ are the system output and input signals, the (Nxm)-matrix $\Theta \in R^{N x m}$ contains the unknown parameters to be estimated, the regressor matrix $\Phi(x(t)) \in R^{N}$ represents the $\mathrm{N}$-dimensional vector of chosen basis functions and $\varepsilon(t) \in R^{m}$ denotes non-parametric uncertainty
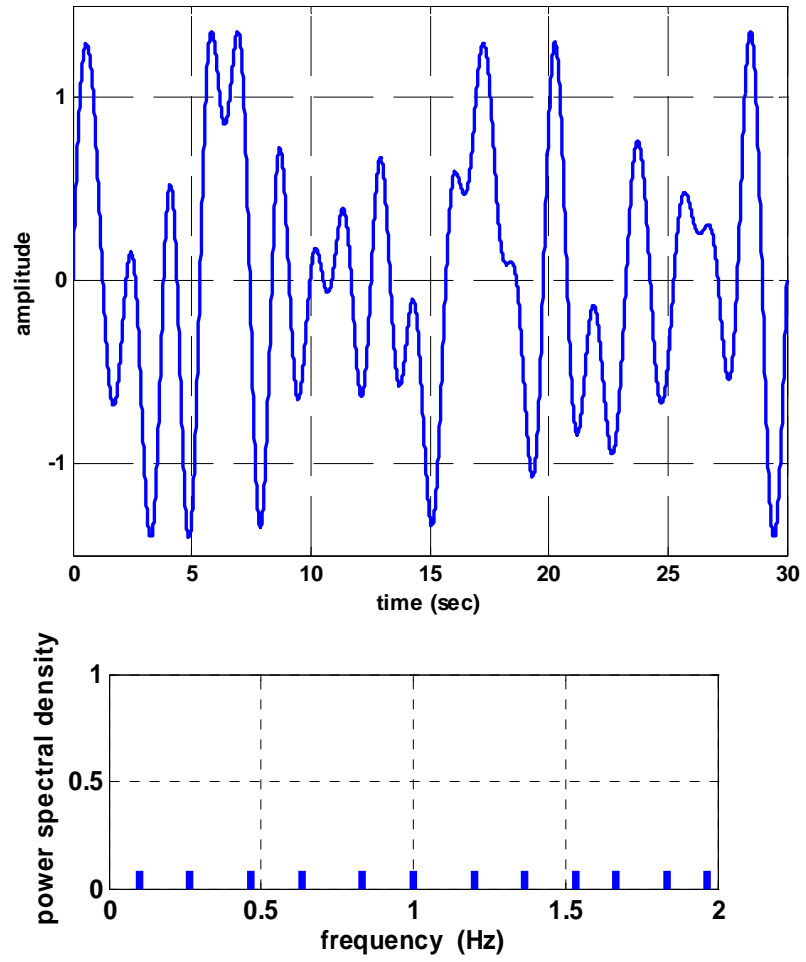

Figure 1. Longitudinal input signal.

in the system (such as noise, modeling error, etc.). If $\Phi(x(t))$ is chosen to be a Radial Basis Function (RBF), then this formulation exploits the Universal Approximation properties of the RBFs covered by the Michelli's theorem, which assures that it is always possible to approximate $f(x)$ on a grid of points using $\hat{f}(x)=\sum_{i}^{N} \theta_{i} \phi\left(x-x_{i}\right)$ such that $f\left(x_{i}\right)=\hat{f}\left(x_{i}\right)$ for all $\left\{x_{i}\right\}_{i=1}^{N}$.

In many practical applications, the RBFs are Gaussian functions of the form:

$$
\phi_{i}(x)=e^{\frac{\left\|x-C_{i}\right\|^{2}}{2 \sigma_{i}^{2}}}, \quad(i=1, \ldots, N)
$$

where $\sigma_{i}$ represents the width and $C_{i} \in R^{n}$ is the center of the $i$ th Gaussian function and the regressor vector is

$$
\Phi(x)=\left(\phi_{1}(x) \ldots \phi_{N}(x)\right)^{T} \in R^{N} .
$$

In on-line estimation, the non-parametric uncertainty $\varepsilon(t)$ is omitted and the estimated value $\hat{\Theta}=\hat{\Theta}(t)$ is updated once a new set of data $y(t)$ and $\Phi(x(t))$ becomes available. 


\section{Gradient Descent Estimator ${ }^{21}$}

Assume that the output of the model (3) is scalar and there are no non-parametric uncertainties in the model; that is, $\varepsilon(t)=0$ and the static linear in parameters model takes the form

$$
y(t)=\theta^{T} \Phi(x(t)) .
$$

Let $\hat{\theta}(t)$ denote the parameter estimation vector at time $t$. Then the output prediction is of the form

$$
\hat{y}(t)=\hat{\theta}^{T} \Phi(x(t)) .
$$

The basic idea in the gradient based estimation is to update the estimated parameters $\hat{\theta}(t)$ in such a way that the prediction error

$$
e_{y}=\hat{y}(t)-y(t)
$$

is reduced. The parameter estimation proceeds in the inverse direction of the gradient of the weighted squared prediction error with respect to the estimated parameters. The online implementation of the Gradient Estimator (GDE) is of the form

$$
\dot{\hat{\theta}}(t)=-\Gamma \Phi(x(t))\left(\Phi(x(t))^{T} \hat{\theta}(t)-y(t)\right)
$$

where $\Gamma=\Gamma^{T}>0$ is a symmetric positive definite matrix called the estimation gain and $\hat{y}(t)=\Phi(x(t))^{T} \hat{\theta}(t)$ represents the predicted/estimated output of the model at time $t$. A common choice for $\Gamma$ is $\Gamma=\gamma I_{N x N}$ where $\gamma$ is a positive scalar gain. Increasing $\gamma$ leads to faster estimation and prediction but at some point larger size leads to oscillatory behavior and slower convergence and increased sensitivity to noise and disturbances. One potential issue with GDE is slow convergence; thus, multiple estimation algorithms should be applied to the data.

\section{Least Squares Estimator with Exponential Forgetting ${ }^{21}$}

Another approach to estimation is least-squares minimization. In this approach, instead of minimizing instantaneous prediction error $e_{y}=\hat{y}(t)-y(t)$, a quadratic cost function of the prediction error with respect to $\hat{\theta}(t)$ is minimized. This Least-Squares Estimator is characterized by good robustness with respect to noise and disturbances, but poor ability in tracking time-varying parameters. The reason for the latter is that the least-squares estimate attempts to fit all the data up to the current time while, in reality, the old data is generated by old parameters and thus should be discounted when estimating the current parameters.

Exponential forgetting of data is a very useful technique in dealing with time-varying unknown parameters. Hence, instead of penalizing all of the past errors from $\tau=0$ to $\tau=\mathrm{t}$ that are due to $\hat{\theta}(t) \neq \theta$ (as it is done when deriving the pure Least-Squares Estimator), the minimization problem is posed for the cost function with the socalled exponential forgetting factor $\beta(t, \tau)=\exp \left[-\int_{\tau}^{t} \lambda(r) d r\right]$. If $\lambda(r)=\lambda \geq 0$ is a constant, then $\beta(t, \tau)=e^{-\lambda(t-\tau)}$. This data forgetting feature of the Least-Squares Estimator with Exponential Forgetting (LSEEF) results in the estimator ability to track slowly varying parameters.

The recursive online implementation of the LSEEF is given by

$$
\begin{array}{ll}
\dot{\hat{\theta}}(t)=-\Gamma(t) \Phi(x(t))\left(\Phi(x(t))^{T} \hat{\theta}(t)-y(t)\right), & \hat{\theta}(0)=\hat{\theta}_{0} \\
\dot{\Gamma}(t)=\lambda(t) \Gamma(t)-\Gamma(t) \Phi(x(t)) \Phi(x(t))^{T} \Gamma(t), & \Gamma(0)=\Gamma_{0}
\end{array} .
$$

Note that LSEEF has the ability to track time-varying parameters but there is a possibility of gain windup in the absence of persistency of excitation. The gain windup problem can be overcome by employing the bounded gain forgetting technique. In the case presented here, the pilot was constantly involved in the tracking task and gain windup never became an issue. However, especially if the pilot is only intermittently involved in hands on the stick, bounded gain forgetting modification will become necessary in future tasks.

\section{Results}

The effects of individual subject characteristics and the vehicle dynamics on the ability of GDE and LSEEF to estimate Stick_hat, the estimated position of the stick $(\hat{y}(t)$ in the discussion above), are presented below. In this case, the preferred outcome of the analyses would be for there to be no differences in the ability of the estimator algorithms with respect to the subject and the vehicle dynamics. This would indicate that the accuracy of the 
estimation methods is not a function of individual subjects or the specific vehicle characteristics; thus, the estimator algorithms may have the capability to be applied broadly.

Additionally, Stick_hat was estimated using two different input functions ( $x(t)$ in the discussion above): (1) the commanded pitch position, Pitch_cmd and (2) the error between actual vehicle pitch and Pitch_cmd (Pitch Pitch_cmd). Obviously, other functions are possible but these two represent basic methods of tracking a driving function, pursuit and compensatory.

Lastly, the ability of the GDE and LSEEF methods to estimate Stick hat using data from a more realistic flight maneuver was examined. This data also contained a change in aircraft dynamics due to surface failures and it was of interest to briefly investigate how well the algorithms would handle these changes in dynamics.

\section{A. Effects of Subjects and Vehicle Dynamics on Estimator Methods}

In order to determine if either the GDE or LSEEF methods to calculate Stick_hat were affected by the vehicle dynamics or individual subject characteristics, an analysis of variance was done with subject and vehicle dynamics as the independent variables and the mean of the absolute difference between Sitck and Stick hat $\left(\mu_{|\delta-\hat{\delta}|}\right)$ as the dependent variable. Significance set at $\mathrm{p} \leq 0.05$. Analyses indicate that neither subject nor the vehicle dynamics statistically significantly affected $\mu_{|\delta-\hat{\delta}|}$ for both the GDE and LSEEF methods for calculating Stick_hat. This implies that both methods to calculate Stick_hat were equally accurate irrespective of the vehicle dynamics over the tested ranges listed in Table 1. Furthermore, these methods showed promise in accounting for individual subject manual control differences. This suggests that the GDE and LSEEF methods to calculate Stick_hat do not require any "tuning" with respect to vehicle dynamics and subject characteristics.

Statistical significance was also not reached for $\mu_{|\delta-\hat{\delta}|}$ when Stick_hat was calculated from Pitch_cmd or Pitch-Pitch_cmd $\left(e\left(\theta-\theta_{\text {cmd }}\right)\right)$ although the GDE method was slightly better calculating Stick_hat using Pitch_cmd (see Fig. 2). Therefore, the inputs Pitch_cmd or $e\left(\theta-\theta_{c m d}\right)$ are equally accurate for calculating Stick_hat.

As mentioned above, subject was not statistically significant for $e\left(\theta-\theta_{c m d}\right)$ when comparing the GDE and LSEEF methods. However, looking at the Stick tracking errors for both methods by signal input, the GDE method was slightly more accurate for subjects when the tracking signal was Pitch_cmd (see Fig. 3) whereas for the LSEEF method, the accuracy of using either Pitch or $e\left(\theta-\theta_{\text {cmd }}\right)$ was essentially equal for all subjects (see Fig. 4). Therefore, it may be possible to tease out some piloting characteristic differences when using the GDE method to estimate/predict Stick_hat.

\section{B. Stick_hat Estimation Differences between Estimator Methods}

Not surprisingly, the GDE method is faster than the LSEEF method even though the accuracy of Stick_hat for these dynamics and control task were basically the same for both methods (see above). The typical time to calculate a $32 \mathrm{sec}$ run for the GDE method was around a second and the typical time for the LSEEF method was just under 6 seconds.

An example of the accuracy of the GDE method to calculate Stick_hat from Pitch_cmd and $e\left(\theta-\theta_{\text {cmd }}\right)$ is shown in Figure 5. For that subject's run, $\mu_{|\delta-\hat{\delta}|}$ when using Pitch_cmd was $0.068(3.4 \%$

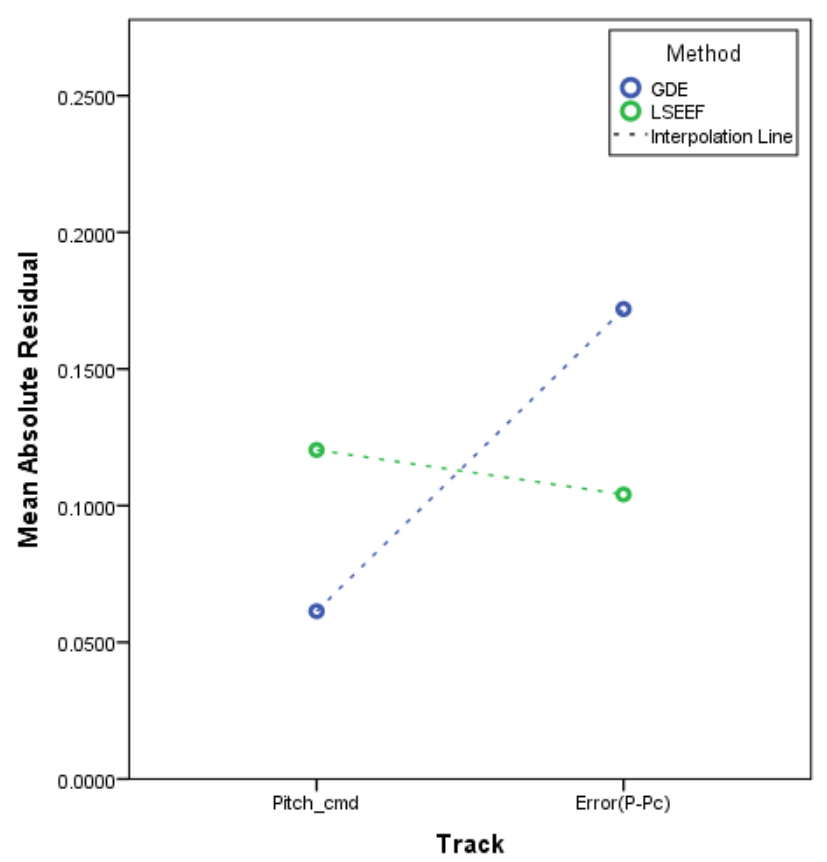

Figure 2. $\mu_{|\delta-\hat{\delta}|}$ as a function of Estimation Method

(GDE or LSEEF) and Input Signal (Pitch_cmd or

$$
\left.e\left(\theta-\theta_{\text {cmd }}\right)\right) \text {. }
$$


of total possible stick throw) and 0.050 (2.5\% of total possible stick throw) when using $e\left(\theta-\theta_{\text {cmd }}\right)$.

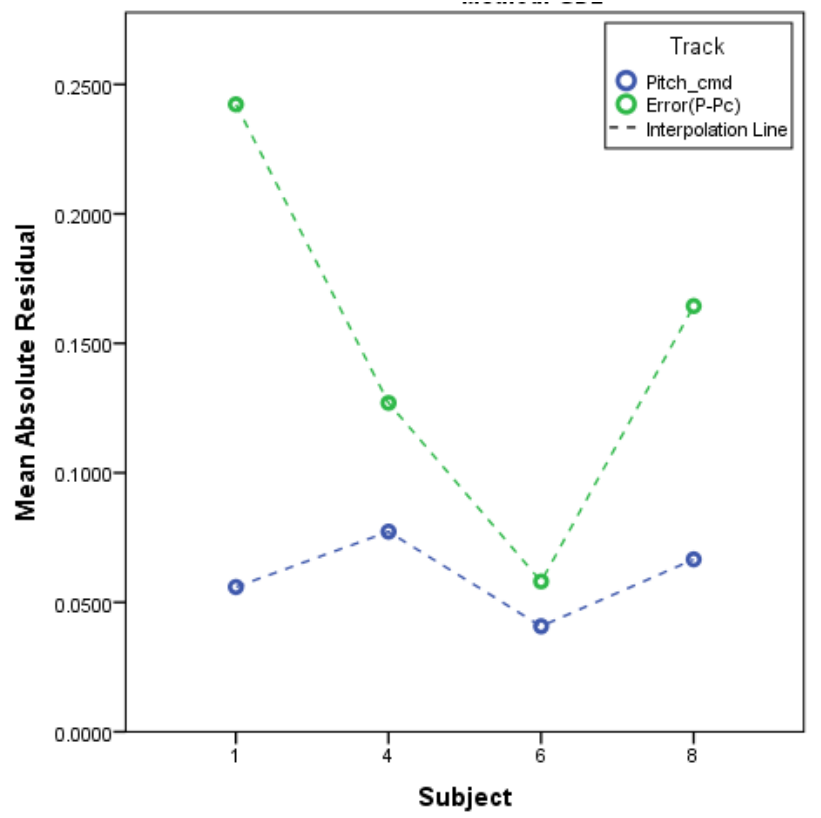

Figure 3. $\mu_{|\delta-\hat{\delta}|}$ as a function of Input Signal

(Pitch_cmd or $\left.e\left(\theta-\theta_{\text {cmd }}\right)\right)$ and Subject for the GDE method.

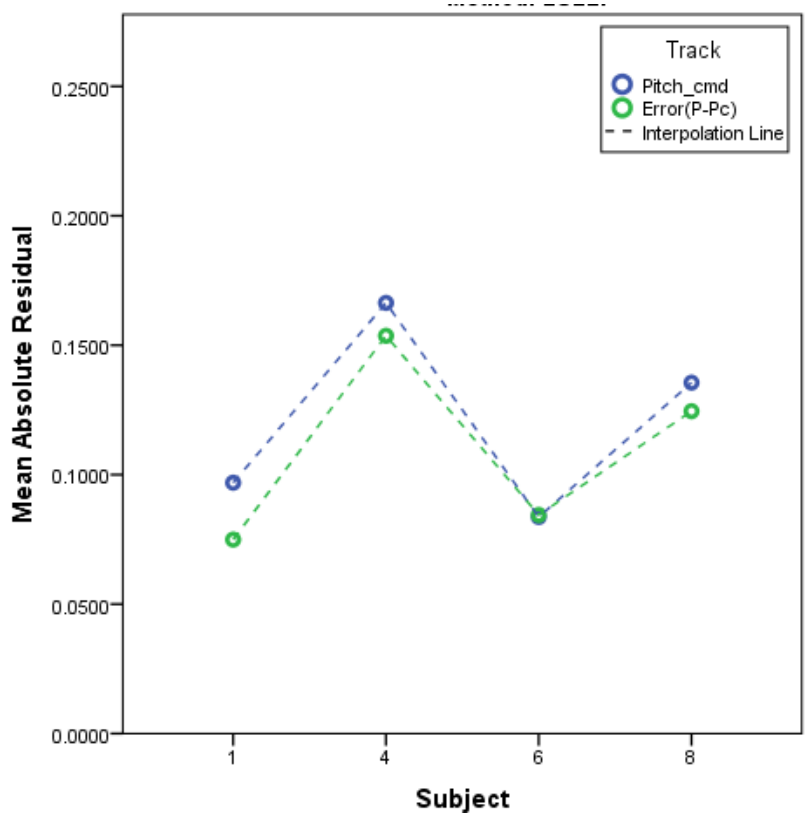

Figure 4. $\mu_{|\delta-\hat{\delta}|}$ as a function of Input Signal (Pitch_cmd or $\left.e\left(\theta-\theta_{\text {cmd }}\right)\right)$ and Subject for the LSEEF method.
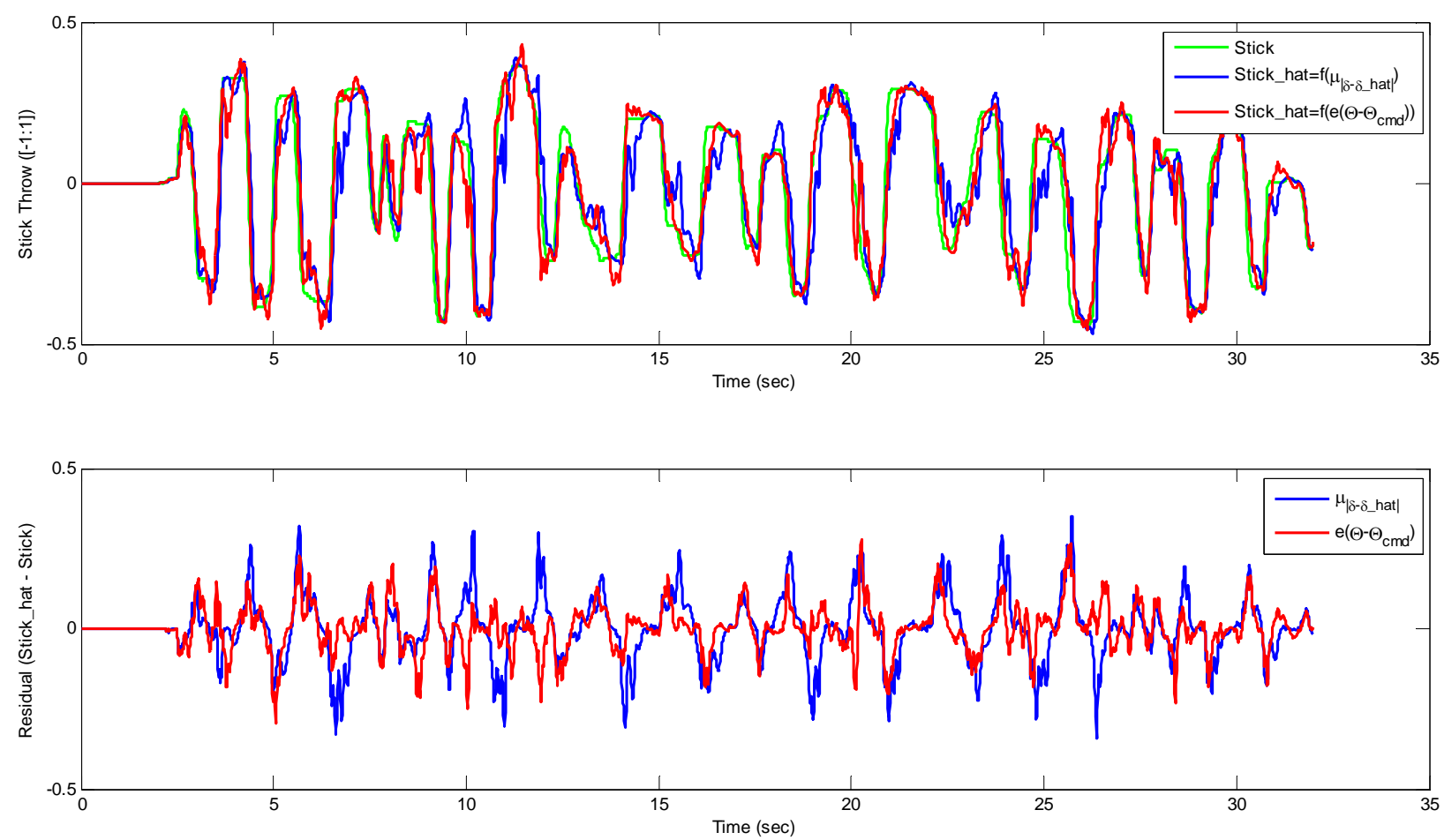

Figure 5. Stick and Stick_hat with Pitch_cmd and $e\left(\theta-\theta_{\text {cmd }}\right)$ and their associated residuals as the input for the GDE method for a particular subject's run.

6

American Institute of Aeronautics and Astronautics 
An example of the accuracy of the LSEEF method to calculate Stick_hat from Pitch_cmd and $e\left(\theta-\theta_{\text {cmd }}\right)$ is shown in Figure 6. For that subject's run, $\mu_{|\delta-\hat{\delta}|}$ when using Pitch_cmd was 0.11 (5.6\% of total possible stick throw) and 0.09 (4.5\% of total possible stick throw) when using $e\left(\theta-\theta_{\text {cmd }}\right)$.
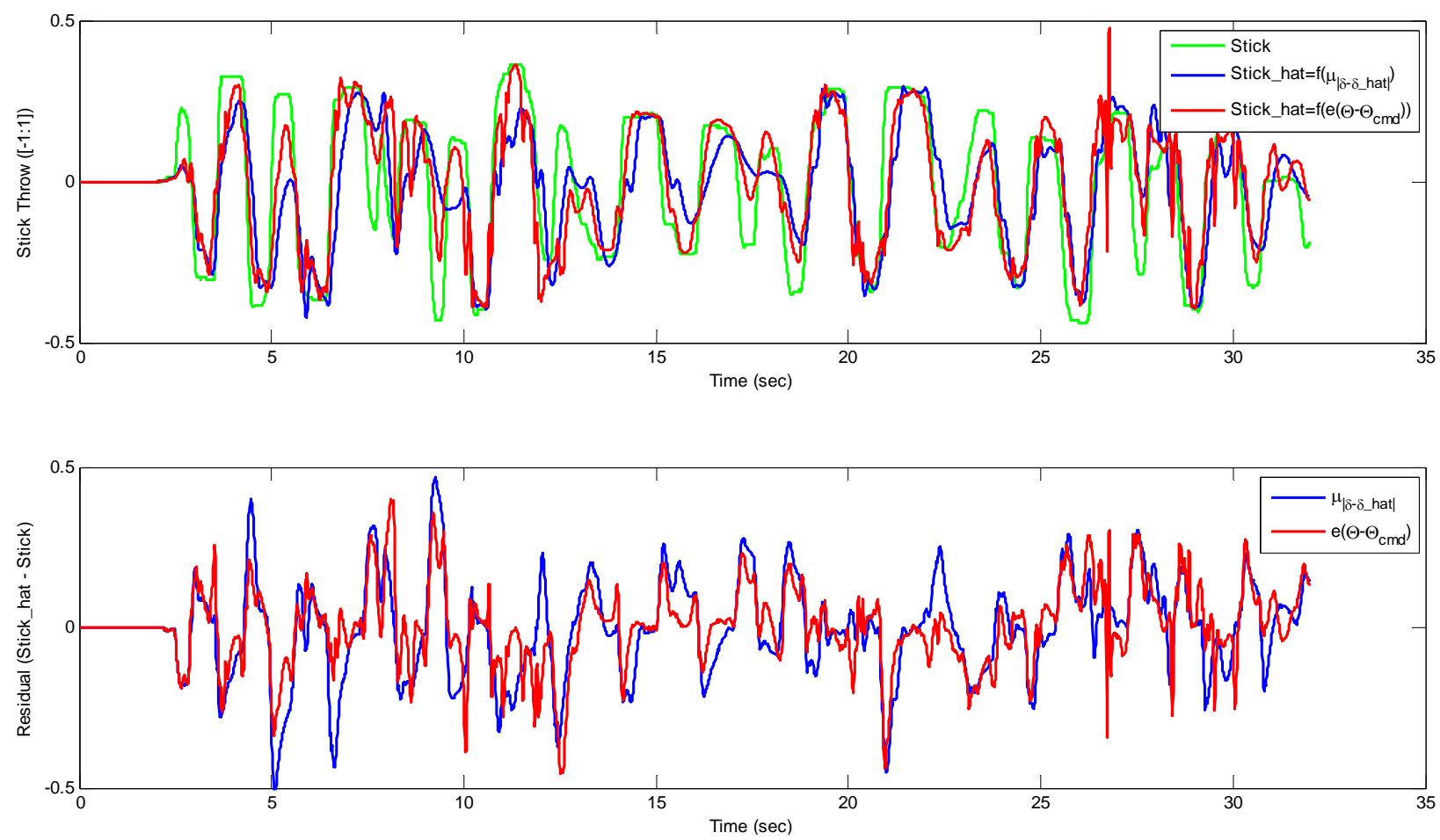

Figure 6. Stick and Stick_hat with Pitch_cmd and $e\left(\theta-\theta_{c m d}\right)$ and their associated residuals as the input for the LSEEF method for a particular's subject run.

\section{Detecting Changing Vehicle Dynamics}

Because both the GDE and LSEEF methods to estimate Stick_hat were successful, data from another experiment that involved changing vehicle dynamics were used to see if Stick_hat could be successfully estimated and to investigate the effects of the changing dynamics on the accuracy of Stick_hat. This experiment involved having subjects again follow the flight director for $90 \mathrm{sec}$ at cruise altitude and speed during either a $2000 \mathrm{ft} / \mathrm{min}$ climb or descent, or during a heading change. During this time, the vehicle dynamics changed from nominal because of stuck control surfaces. The control system used by this experiment was an $\mathcal{L}_{1}$ adaptive control system that would either adapt immediately, in $3 \mathrm{sec}$, in $7 \mathrm{sec}$, or never. The GDE method was used to analyze the longitudinal data because of the computation times and possible gain windup discussed above.

For a subject's ability to track longitudinally, the GDE method was able to successfully estimate Stick_hat using both the Pitch and $e\left(\theta-\theta_{c m d}\right)$ input signals (see Figs. 7 and 8). The change in dynamics is visible when analyzing $\mu_{|\delta-\hat{\delta}|}$. When the adaptive controller does not compensate for the surface failures, $\mu_{|\delta-\hat{\delta}|}$ when using Pitch as the input from a particular subject's run went from 0.006 before the failure to 0.012 after the failure (see Table 2 and Fig. 7). When using $e\left(\theta-\theta_{c m d}\right)$ as the input, $\mu_{|\delta-\hat{\delta}|}$ for a particular subject's run went from 0.009 before the failure to 0.034 after the failure. When the adaptive controller immediately compensated for the stuck surfaces, the average absolute error before the failure, during the failure $+3 \mathrm{sec}$, and after the failure $+3 \mathrm{sec}$ were essentially the same (see Table 2 and Fig. 8) for a particular subject's $\mu_{|\delta-\hat{\delta}|}$ but $e\left(\theta-\theta_{c m d}\right)$ did show an increase during the failure $+3 \mathrm{sec}$. The $3 \mathrm{sec}$ were added in order to account for the time it typically takes a human to adapt to changing dynamics $^{22,23}$. As when the adaptive controller adapted immediately, when the adaptation time was 3 or $7 \mathrm{sec}$, the 
Table 2. Effects of changing aircraft dynamics on calculating Stick_hat for a particular subject's run.

\begin{tabular}{|c|c|c|c|c|c|c|c|}
\hline \multirow{2}{*}{$\begin{array}{c}\text { Failure } \\
\text { Onset } \\
\text { Time } \\
\text { (sec) }\end{array}$} & \multirow{2}{*}{$\begin{array}{l}\text { Adaptation } \\
\text { Time (sec) }\end{array}$} & \multicolumn{3}{|c|}{$\mu_{|\delta-\hat{\delta}|}$ for Pitch_cmd } & \multicolumn{3}{|c|}{$\mu_{|\delta-\hat{\delta}|}$ for $e\left(\theta-\theta_{c m d}\right)$} \\
\hline & & $\begin{array}{l}\text { Before } \\
\text { Failure }\end{array}$ & $\begin{array}{l}\text { During } \\
\text { Failure }\end{array}$ & $\begin{array}{c}\text { After } \\
\text { Adaptation }\end{array}$ & $\begin{array}{l}\text { Before } \\
\text { Failure }\end{array}$ & $\begin{array}{l}\text { During } \\
\text { Failure }\end{array}$ & $\begin{array}{c}\text { After } \\
\text { Adaptation }\end{array}$ \\
\hline 12 & Never & 0.006 & 0.012 & & 0.009 & 0.034 & \\
\hline 9 & 3 & 0.001 & 0.002 & 0.001 & 0.001 & 0.006 & 0.002 \\
\hline 21 & 7 & 0.004 & 0.006 & 0.006 & 0.012 & 0.024 & 0.018 \\
\hline 22 & Immediate & 0.005 & 0.005 & 0.007 & 0.006 & 0.013 & 0.007 \\
\hline
\end{tabular}
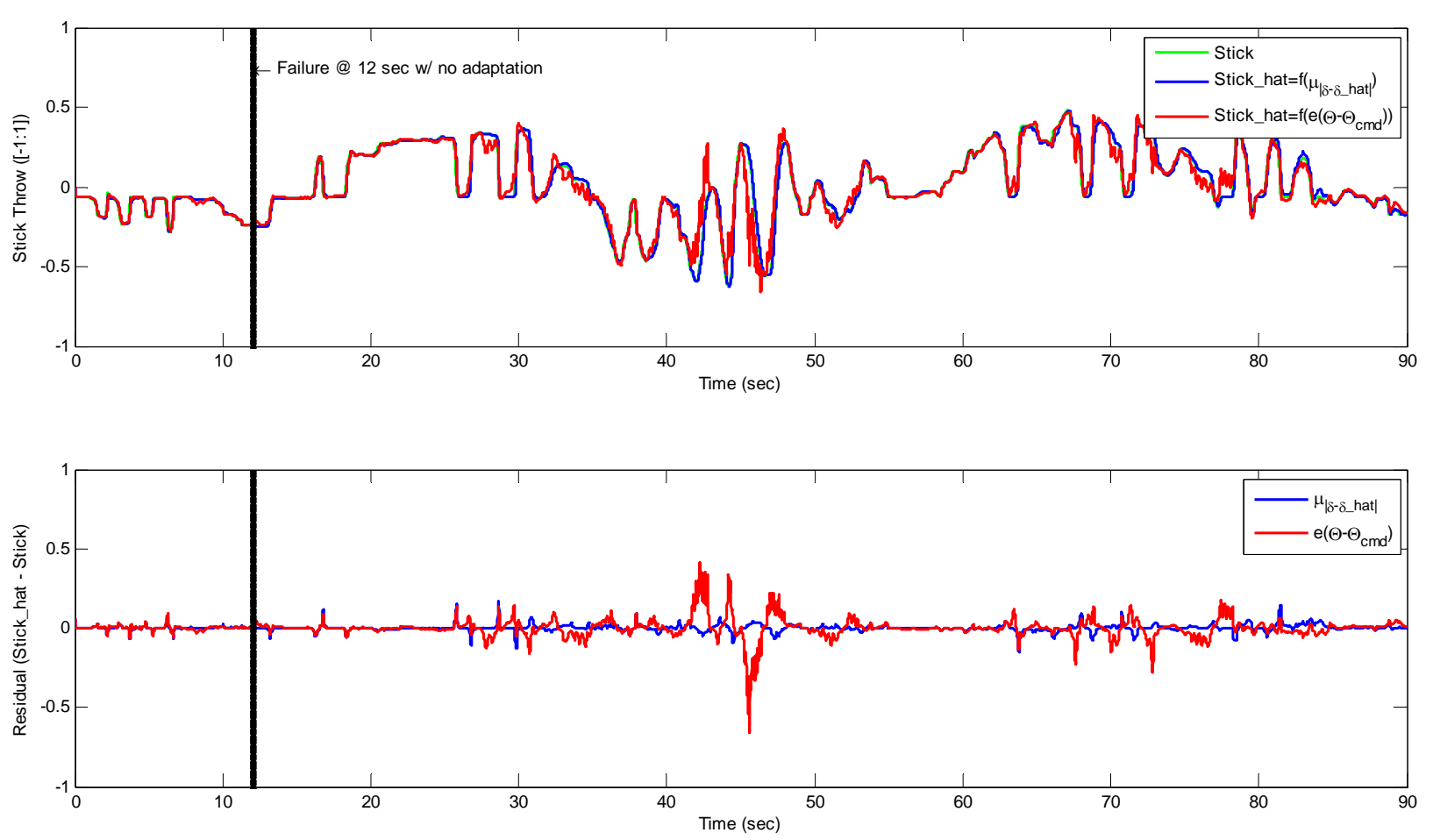

Figure 7. Stick and Stick_hat with Pitch_cmd and $e\left(\theta-\theta_{c m d}\right)$ and their associated residuals as the input for the GDE method with changing aircraft dynamics and no adaptation for a particular subject's run.

$e\left(\theta-\theta_{\text {cmd }}\right)$ input was more sensitive to the subject's adapting to the failure and then to the changes the adaptive controller implemented. As can be seen in Table 2 for a particular subject's $e\left(\theta-\theta_{c m d}\right)$, the "during adaptation after the failure" $\mu_{|\delta-\hat{\delta}|}$ was approximately double the amount as it was both "before failure" and "after adaptation," which included adaptation by both the aircraft control system and the subject.

The apparent ability of $e\left(\theta-\theta_{\text {cmd }}\right)$ in the GDE method to accurately estimate stick position during typical cruise flight maneuvers is promising for possibly detecting changes in pilot behavior, whether these changes are due to changing aircraft dynamics or due to effects on the pilot such as high workload, situation awareness, fatigue, etc.

\section{Discussion}

The purpose of the research described above was to go beyond pilot identification based on collected experimental data and to develop a predictor of pilot behavior. An experiment was conducted to quantify the effects of changing aircraft dynamics on an operator's ability to track a signal in order to eventually model a pilot adapting to changing aircraft dynamics. GDE and LSEEF algorithms used these data to predict pilot stick input.

The results indicate that individual pilot characteristics did not affect the accuracy of the GDE and LSEEF methods to estimate pilot stick input. Therefore, minimal tuning with regards to the pilot will be needed when trying to predict Stick_hat with either methods. Furthermore, the vehicle dynamics also did not affect the accuracy of the 

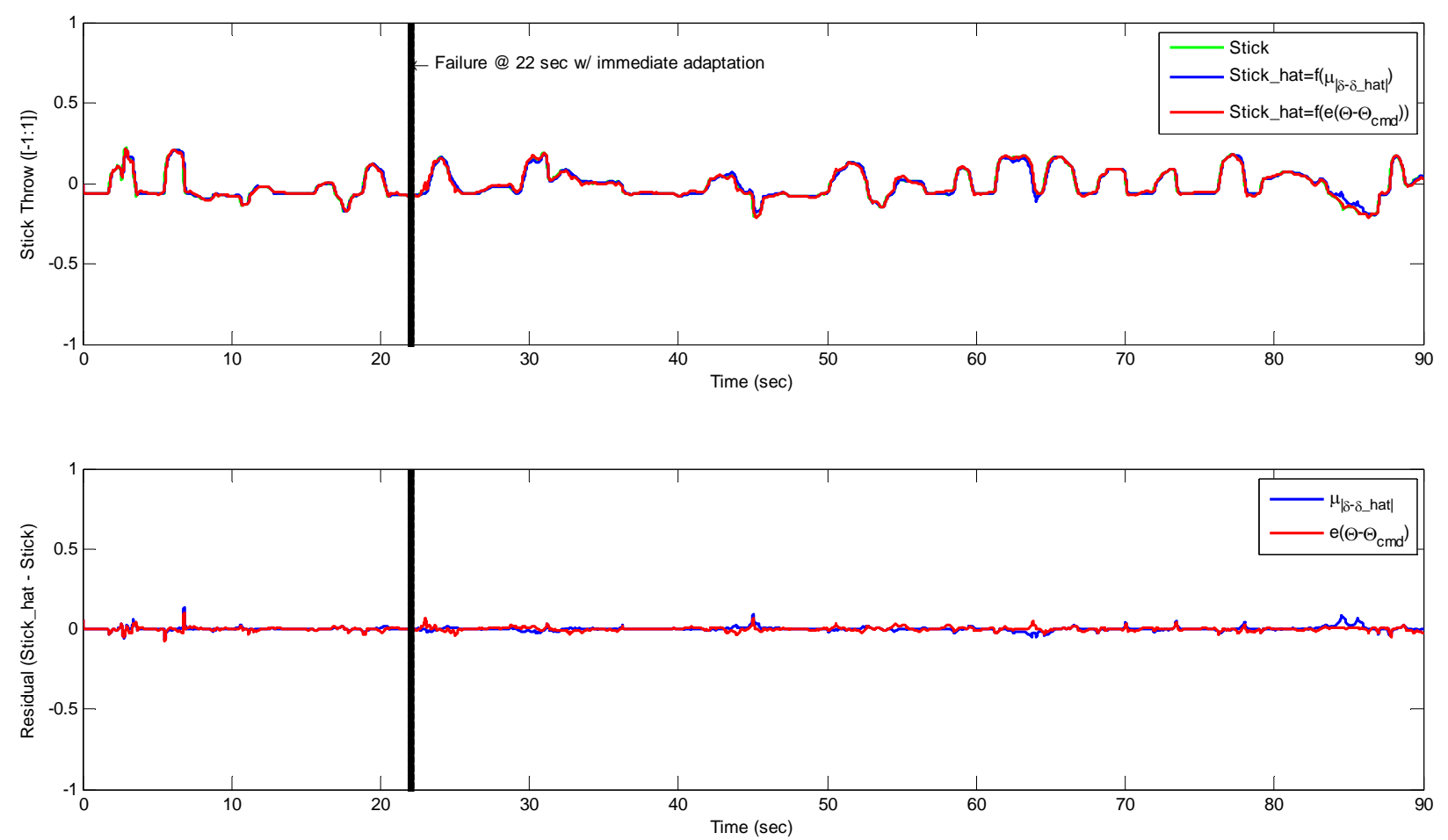

Figure 8. Stick and Stick_hat with Pitch_cmd and $e\left(\theta-\theta_{\text {cmd }}\right)$ and their associated residuals as the input for the GDE method with changing aircraft dynamics and immediate adaptation for a particular subject's run.

two methods to estimate pilot stick input. This again suggests that the methods may not require significant tuning to individual aircraft dynamics.

The methods may also be able to handle changing dynamics; in fact, this was the case. Using driving functions that incorporated changing aircraft dynamics, the GDE was able to accurately estimate pilot stick input before, during, and after a control surface failure that affected the dynamics of the aircraft. In fact, the GDE method was able to accomplish this during basic cruise flight maneuvers that did not entail a great deal of dynamic input by the subject during a manual flight condition. The GDE method may also have the capability to detect a change in the subject's input when looking at the error between the commanded pitch attitude and the vehicle's actual pitch attitude. While the subject appeared to be adapting to the changed aircraft dynamics, this error increased before it settled back down to pre-failure error levels.

Both the GDE and LSEEF methods were equally able to predict the subject's input. However, looking at the stick tracking errors for both methods by signal input, the GDE method was slightly more accurate for subjects when the tracking signal was Pitch_cmd. Therefore, it may be possible to differentiate changes in piloting characteristics due to workload, situation awareness, engagement, etc., when using the GDE method to calculate Stick_hat.

\section{Conclusion}

Although these results are just starting to quantify the abilities of estimator methods to predict pilot stick input, the above results do hint at some capabilities. These capabilities include a minimal need to optimize the algorithms to pilot characteristics or vehicle dynamics, an ability to detect changes in either vehicle dynamics or the state of the pilot, and the capability to predict pilot stick input in real time. The ability of the algorithms to work in real time and their accuracy in predicting pilot stick input may be affected during maneuvers in both longitudinal and lateral directions. Lastly, other input functions may even predict pilot stick input better or detect changes in the pilot or the vehicle.

\section{Acknowledgments}

The authors would like to thank Lucas Hempley of Northrop Grumman for his simulation programming of the experiments that provided the data used in this paper. The authors would also like to thank Chris Hartman for his post-processing of the data and Dr. Gene Morelli of NASA Langley Research Center for his insights into system 
identification. In addition, the authors would like to thank Dr. Eugene Lavretsky of the Boeing Co. for his insights into model estimation and his class notes for CDS 270.

\section{Disclaimer}

The use of trademarks or names of manufacturers in the report is for accurate reporting and does not constitute an official endorsement, either expressed or implied, of such products or manufacturers by the National Aeronautics and Space Administration.

\section{References}

${ }^{1}$ Yilmaz, D., Jump, M., Linghai, L., and Jones, M. "Aircraft and Rotorcraft Pilot Couplings - Tools and Techniques for Alleviation and Detection." Vol. ACPO-GA-2010-266073, Airstotel, 2010, p. 35.

${ }^{2}$ Zaychik, K. B., Cardullo, F. M., and George, G. "A Conspectus on Operator Modeling: Past, Present and Future," Modeling and Simulation Technologies Conference and Exhibit. Vol. AIAA-2006-6625, Keystone, CO, 2006, p. 17.

${ }^{3}$ McRuer, D. T., and Jex, H. R. "A Review of Quasi-Linear Pilot Models," IEEE Transactions on Human Factors in Electronics Vol. HFE8, No. 3, 1967, pp. 231-249.

${ }^{4}$ McRuer, D. T., and Krendel, E. S. "Mathematical models of human pilot behavior." 1974.

${ }^{5}$ Kleinman, D. L., Baron, S., and Levison, W. H. "An Optimal Control Model of Human Response|Part 1: Theory and Validation," Automatica Vol. 6, No. 3, 1970, pp. 357-369.

${ }^{6}$ Hess, R. A. "Unified Theory for Aircraft Handling Qualities and Adverse Aircraft-Pilot Coupling," J. Guidance, Control, and Dynamics Vol. 20, 1997, pp. 1141-1148.

${ }^{7}$ Davidson, J. D., and Schmidt, D. K. "Modified Optimal Control Pilot Model for Computer-Aided Design and Analysis." Vol. NASA-TM-4384, NASA, 1992.

${ }^{8}$ Schmidt, D. K. "Optimal Flight Control Synthesis Via Pilot Modeling," J. Guidance \& Control Vol. 2, No. 4, 1979 , pp. $308-312$.

${ }^{9}$ Hess, R. A. "Simplified approach for modeling pilot pursuit control behaviour in multi-loop flight control tasks," Proceedings of the Institution fo Mechanical Engineers, Vol. 220 Part G: Journal of Aerospace Engineering, 2006, pp. 85-102.

${ }^{10}$ Hess, R. A. "A Preliminary Study of Human Pilot Dynamics in the Control of Time-Varying Systems," AIAA Modeling and Simulation Technologies Conference. Vol. AIAA-2011-6554, Portland, OR, 2011.

${ }^{11}$ Brieger, O., Ossmann, D., and Rüdinger, M. "A New Flight Test Technique for Pilot Model Identification," AIAA Atmospheric Flight Mechanics Conference and Exhibit. Vol. AIAA-2008-6556, Honolulu, HI, 2008, p. 16.

${ }^{12}$ Lampton, A., and Klyde, D. "Power Frequency - A New Metric for Analyzing Pilot-in-the-Loop Flying Tasks," Atmospheric Flight Mechanics Conference. Vol. AIAA-2011-6539, AIAA, Portland, OR, 2011, p. 21.

${ }^{13}$ Thompson, P., Bachelder, E., Klyde, D., Harris, C., and Brenner, M. "Wavelet-Based techniques for Improved Online Systems Identification," USAF Developmental Test and Evaluation Summit. Vol. AIAA-2004-6838, AIAA, Woodland Hills, CA, 2004.

${ }^{14}$ Thompson, P., Klyde, D., and Brenner, M. "Wavelet-Based Time-Varying Human Operator Models," Atmospheric Flight Mechanics Conference and Exhibit. Vol. AIAA-2001-4009, AIAA, Montreal, Canada, 2001, p. 12.

${ }^{15}$ Zaal, P., Pool, D. M., Mulder, M., and van Paassen, M. M. "Multimodal Pilot Control Behavior in Combined Target-Following Distrubance-Rejection Tasks," AIAA Modeling and Simulation Technologies Conference. Vol. AIAA-2009-6057, Chicago, IL, 2009, p. 18.

${ }^{16}$ Zaal, P., Pool, D. M., Mulder, M., and van Paassen, M. M. "Multimodal Pilot Model Identification in Real Flight," AIAA Modeling and Simulation Technologies Conference. Vol. AIAA-2009-6028, AIAA, Chicago, IL, 2009, p. 20.

${ }^{17}$ Zaal, P., and Sweet, B. "Estimation of Time-Varying Pilot Model Parameters," AIAA Modeling and Simulation Technologies Conference. Vol. AIAA-2011-6474, AIAA, Portland, OR, 2011.

${ }^{18}$ Trujillo, A. C., and Gregory, I. M. "Piloting Changes to Changing Aircraft Dynamics: What Do Pilots Need to Know?," 30th Digital Avionics Systems Conference. IEEE, Seattle, WA, 2011.

${ }^{19}$ Klein, V., and Morelli, E. A. Aircraft System Identification Theory and Practice: AIAA, 2006.

${ }^{20}$ Morelli, E. A. "Flight Test Experiment Design for Characterizing Stability and Control of Hypersonic Vehicles," AIAA-20081682, U.S. Air Force T\&E Days. Los Angelos, CA, 2008.

${ }^{21}$ Lavretsky, E. "CDS Lecture Notes - Spring 2008." California Institute of Technology, Pasadena, CA, 2008.

${ }^{22}$ Delp, P., and Crossman, E. R. F. W. "Transfer Characteristics of Human Adaptive Response to Time-Varying Plant Dynamics." 1972.

${ }^{23}$ Hess, R. A. "Modeling Pilot Control Behavior with Sudden Changes in Vehicle Dynamics," Journal of Aircraft Vol. 46, No. 5, 2009, pp. 1584-1592. 